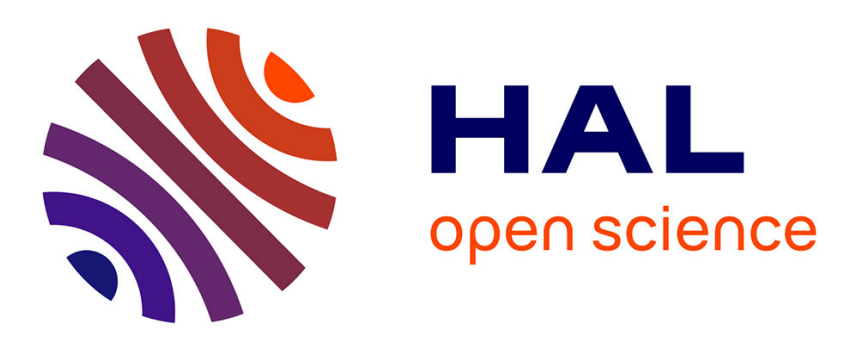

\title{
Patrimonialisation des savoir-faire et résidences d'artistes : un dispositif atypique de collection d'entreprise
}

\author{
Véronique Anderlini-Pillet
}

\section{- To cite this version:}

Véronique Anderlini-Pillet. Patrimonialisation des savoir-faire et résidences d'artistes: un dispositif atypique de collection d'entreprise. Recherches en communication, 2018, 45, 10.14428/rec.v45i45 . hal-03519296

\section{HAL Id: hal-03519296 \\ https://hal.science/hal-03519296}

Submitted on 10 Jan 2022

HAL is a multi-disciplinary open access archive for the deposit and dissemination of scientific research documents, whether they are published or not. The documents may come from teaching and research institutions in France or abroad, or from public or private research centers.
L'archive ouverte pluridisciplinaire HAL, est destinée au dépôt et à la diffusion de documents scientifiques de niveau recherche, publiés ou non, émanant des établissements d'enseignement et de recherche français ou étrangers, des laboratoires publics ou privés. 


\title{
Patrimonialisation des savoir-faire et résidences d'artistes : un dispositif atypique de collection d'entreprise
}

\author{
Véronique Anderlini-Pillet \\ Docteur en SIC \\ Chercheuse associée \\ SIC.Lab Méditerranée \\ Université Côte d'Azur
}

\section{Résumé}

Au cœur de «l'économie de l'enrichissement» décrite par Luc Boltanski et Arnaud Esquerre dans Enrichissement, une critique de la marchandise (2017) la pratique de la collection a acquis une dimension communicationnelle centrale pour les entreprises, notamment dans le secteur du luxe. Le travail présenté ici résulte de l'approfondissement d'un élément de notre thèse réalisée en 2016 ayant pour sujet l'identification des mécanismes d'artification du luxe dans le discours médiatique. Nous nous sommes focalisée ici sur une seule des vingt-cinq entreprises que nous avions étudiées, le groupe Hermès et son dispositif de communication constitué par les résidences d'artistes au cœur de sa fondation d'entreprise. Nous avons cherché à déterminer si l'imbrication complexe entre métier et art, au carrefour de l'artification (N. Heinich \& R. Shapiro, 2012) et des savoir-faire, fait apparaître un nouveau type de collection d'entreprise qui allie racines historiques, métiers ancestraux et pratiques actuelles de l'art contemporain.

\section{Mots clés}

Artification, patrimonialisation, Fondation Hermès, création, luxe.

Au cœur de «l'économie de l'enrichissement» décrite par Luc Boltanski et Arnaud Esquerre dans leur ouvrage Enrichissement, une critique de la marchandise (2017), la pratique de la collection a acquis une dimension communicationnelle centrale pour les entreprises « permettant de mettre en rapport les différents domaines d'activités marchandes sur lesquels repose ce type d'économie» (L. Boltanski \& A. Esquerre, 2017, p. 48). Dans cette «économie des choses enrichies » (Ibid.), l'industrie du luxe tente de maintenir par la 
communication une différenciation au moins symbolique avec d'autres marchandises industrielles.

L’industrie du luxe est caractérisée par Lionel Fontagné comme celle des entreprises qui ont réussi « à la frontière de l'artisanat haut de gamme traditionnel à maintenir les marques européennes et un savoir-faire dans les secteurs pourtant décimés par la concurrence des pays à bas coût de main d'œuvre» (L. Fontagné, 2013, p. 3). D'autres chercheurs accusent l'industrie du luxe d'avoir tué le luxe à la fin du $X^{\mathrm{e}}$ siècle (M. de Ferrière Le Vayer, 2007, p.169) en le faisant entrer dans l'ère de la consommation de masse alors que justement la mécanique du désir de luxe fonctionne sur la rareté symbolique et la distinction sociale de celui qui en jouit. Cette industrie produit un storytelling, c'est-à-dire un récit enjolivé de la valeur intrinsèque et symbolique de la production artisanale pour entretenir le mythe du produit d'exception alors qu'elle a intégré tous les procédés de fabrication industrielle et de distribution de masse. Le produit de luxe se définit aussi comme celui que le consommateur est prêt à payer à un prix supérieur à un autre article procurant la même valeur d'usage pour acquérir une jouissance supérieure : un objet de désir «mixant élitisme traditionnel et modernité accessible » (Joël Ravix, 2013, p.47).

La maison Hermès garde une petite activité artisanale dédiée aux commandes spéciales de ses clients les plus exigeants qu'elle met en avant dans sa communication de marque comme un standard généralisé de fabrication mais produit principalement de manière industrielle de la mode, des accessoires des parfums et des objets en cuir emblématiques d'une industrie du luxe qui oscille entre «expériences [sensibles], arrogance [de la distinction sociale], authenticité [de certains produits] » (Y. Michaud, 2013).

Dans notre thèse nous avons étudié les stratégies de communication esthétisée de vingt-cinq entreprises internationales du luxe pour établir une typologie des dispositifs d'artification. La résidence d'artistes en entreprise est un de ces dispositifs mais semble aussi être la matière première d'une forme innovante de collection d'entreprise.

\section{Artification et résidences d'artistes}

Pour analyser la relation entre les résidences d'artistes et la genèse d'une collection d'entreprise nous devons revenir brièvement sur la signification de l'artification en général et de celle à l'œuvre dans le luxe comme étape stratégique de la valorisation du patrimoine de ses entreprises. Le concept d'artification c'est-à-dire «le passage à l'art » d'objets ou de pratiques qui n'appartiennent pas au monde de l'art a été mis en lumière par Nathalie Heinich et Roberta Shapiro dans un ouvrage paru en 2012 (N. Heinich \& R. Shapiro, 2012, p. 19). En recourant à ce nouveau mot pour désigner la transformation de pratiques qui deviennent 
artistiques ou d'objets en œuvres, ces auteurs ont exploré les aspects dynamiques du passage à l'art en se posant la question «Comment l'art vient-il aux personnes, aux objets, aux façons de faire ou de penser? Et comment en retour agit-il ?» (Ibid. 2012, p. 268). Le processus d'artification repose d'abord sur des éléments concrets comme la transformation du contenu et de la forme de l'activité, une reconstruction d'objets nouveaux et un réagencement de dispositifs organisationnels. Dans l'industrie du luxe, ces dispositifs tendent à transformer l'objet en œuvre, le savoir-faire en art et les clients en spectateurs afin de faire advenir un monde de l'art dans un univers marchand.

Hermès est la seule maison de luxe qui a réussi un «passage à l'art » d'un de ses produits lors d'une vente aux enchères de pièces rares. Ce dispositif lui a permis de faire « fonctionner une chose comme une œuvre d'art à un certain moment et non à d'autres... mais seulement quand elle fonctionne symboliquement» comme Nelson Goodman l'écrit dans son article «Quand y-a-t-il art?»(1978). Cette artification réussie dans une entreprise qui se distingue sur d'autres points (historicité, indépendance, préservation du caractère familial et résultats économiques hors normes) nous a décidée à analyser un autre dispositif esthétique de communication de cette maison de luxe : les résidences d'artistes.

\section{Une forme atypique de collection d'entreprise}

Ce procédé d'imbrication entre savoir-faire et création au carrefour de la valorisation de l'identité de marque et du mécénat d'art constitue-t-il une forme atypique de collection d'entreprise?

Nous avons formulé l'hypothèse que son articulation avec les autres dispositifs de patrimonialisation représente une valorisation de la création mais aussi un moyen de gérer la double temporalité entre tradition et projection vers le futur de la marque de luxe et de ses savoir-faire formant ainsi un récit rétro-futuriste imprégné d'une contemporanéité historicisée. Nous avons disposé d'un corpus documentaire constitué de tous les supports de communication d'entreprise existants: le groupe coté en bourse produit des rapports d'activité et de RSE (Responsabilité Sociale et Environnementale), a plusieurs sites internet structurés selon les différentes facettes de l'entreprise (portail général, site institutionnel, galerie en ligne, e-shop, site de la fondation d'entreprise), bénéficie de nombreuses parutions de presse grâce à son accès aux médias facilité par l'importance de son budget publicitaire, et un ensemble de quinze vidéos concernant la genèse des trois résidences d'artistes que nous allons présenter.

Nous nous sommes concentrée sur une analyse comparée des contenus : images, dialogues et des discours dans les vidéos et sur les pages internet dédiés aux résidences d'artistes, en ayant 
déjà visité in situ certains sites concernés et en ayant une connaissance pratique de la plupart des savoir-faire utilisés.

\section{Musée historique versus collection d'entreprise}

Le patrimoine historique constitue un élément récurrent des stratégies de communication de l'industrie du luxe. Il valorise l'ancienneté des maisons par rapport aux entreprises plus récemment créées. Une création au XIX ${ }^{\text {ème }}$ siècle suffit à ancrer définitivement l'histoire de l'entreprise dans l'Histoire, faisant apparaître a contrario les maisons postérieures à la Seconde Guerre Mondiale comme des «nouvelles » maisons moins valorisantes pour leurs clients. En effet, il semble qu'à côté des lignées familiales en déshérence, la lignée du produit ait gagné ses lettres de noblesse avec force marketing élitiste. Dominique Poulot écrit «Progressivement, l'engouement pour la promotion et pour la valorisation du patrimoine fait figure de véritable « croisade » au sein du monde occidental» (D. Poulot, 2006, p. 38). Les entreprises du luxe ont saisi les potentialités communicationnelles de la collection d'entreprise dans le récit historique de la maison tout en mesurant le risque de laisser la marque apparaître comme une relique poussiéreuse pour les nouvelles générations de consommateurs.

Si nous avons formulé l'hypothèse que les résidences d'artistes semblent être le dernier maillon de la chaîne de patrimonialisation de la maison Hermès articulant le passé, le présent et le futur, c'est parce qu'il existe aussi un musée historique comme premier maillon de cette chaine. Le musée d'entreprise décrit par comme une "genre composite... (une) mosaïque » (I. Cousserand, 2012, p. 193), est toujours identifié à un lieu, des pratiques et une histoire. Dans le cas de la maison Hermès la seule entité qui corresponde à ces dispositions est un cabinet de curiosités situé à l'étage du magasin parisien historique du 24 Faubourg Saint-Honoré qui ne se visite que sur invitation. Les stylistes de la maison sont tenus de venir s'y imprégner de «l'esprit » Hermès. Ce musée d'entreprise a été ouvert au lendemain de la guerre 1914-18 dans le bureau d'Émile Hermès, petit-fils de Thierry Hermès créateur de la maison.

Pour la marque Hermès dont l'emblème est une calèche fort peu contemporaine la question de la valorisation du patrimoine s'est posée avec d'autant plus d'acuité que son histoire est au centre du récit de la marque dans toutes ses productions médiatiques. Il lui faut gérer deux temporalités antagonistes (V. Anderlini-Pillet, 2017, p. 88), l'histoire qui débute en 1837 et le renouvellement de la marque au début du $\mathrm{XXI}^{\mathrm{e}}$ siècle. La maison Hermès fait le choix d'un récit dynamisé par les résidences d'artistes mais au cœur des savoir-faire historiques de l'entreprise que sont la sellerie, la maroquinerie et à partir des années 1930, la soie. 


\section{Résidences d'artistes, fondation et manufactures}

Le philosophe Jacques Rancière dans Le partage du sensible (2000) s'est interrogé sur l'agir humain en général et donc sur l'activité professionnelle et la possibilité d'y englober les pratiques artistiques ou bien de les considérer comme des exceptions aux autres pratiques; dans cette perspective, la résidence d'artistes est une manière de partager l'expérience sensible d'une fusion des savoir-faire des métiers et des pratiques artistiques dans une époque où «le capitalisme artiste ou trans-esthétique n'est pas seulement le système qui acclimate dans le monde de l'entreprise, les valeurs ou l'idéologie artiste, il est avant tout celui qui dilate et incorpore dans son fonctionnement même les activités relevant du monde de l'art », (G. Lipovetsky \& J. Serroy, 2013, p. 123).

Dans le modèle de résidence d'artiste de la maison Hermès, l'entreprise délègue à sa fondation le choix d'un thème général pour un cycle de quatre ans, celui dont font partie les résidences d'artistes étudiées a pour titre «Les Mains sans sommeil » et concerne les années 2013-2016. La fondation d'entreprise choisit aussi les manufactures qui participeront au projet parmi tous les ateliers des métiers du groupe Hermès qui comprennent les métiers du cuir maroquinerie et sellerie, de la soie et des textiles, la bijouterie, l'art de vivre, les arts de la table, les parfums et l'horlogerie. Le conseil d'administration de la fondation choisit ensuite trois « parrains » artistes ou directeurs d'institutions auxquels il délègue le choix des trois artistes qui travailleront un an en collaboration étroite avec les artisans dans une manufacture (un site de production) pour réaliser chacun une œuvre originale en ayant carte blanche. Pour l'année 2015, les binômes artistes-parrains sélectionnés sont :

- Une plasticienne diplômée de l'ENSBA-Paris et des Arts Décoratifs de Strasbourg (Io Burgard) qui a travaillé dans l'atelier de maroquinerie de Seloncourt pour créer une malle-banc en cuir avec des accessoires de voyage surdimensionnés en cuir et métal inspirés des outils de la sellerie (sous le parrainage de Jean-Michel Alberola, artiste).

- Un artiste verrier américain (DH $\mathrm{McNabb}$ ) qui a travaillé à la Cristallerie SaintLouis (filiale du groupe Hermès depuis 1995) à Saint-Louis-Lès-Bitche pour concevoir une série de contenants indéfinis avec un procédé d'emprisonnement d'air entre deux couches de verre (sous le parrainage de Richard Fishman, professeur au département consacré à la création verrière d'une université de Boston). 
- Une plasticienne et chorégraphe (Célia Gondol), diplômée de l'ENSBA-Paris et professeur de danse qui a conçu des tissages de soie et de cuivre, les métrages réalisés faisant l'objet d'une installation (sous le parrainage de Ann Veronica Janssens, artiste et professeur à l'ENSBA).

Chaque résidence d'artiste fait l'objet de vidéos qui sont mises en ligne et utilisées dans les expositions et montrées en interne dans les manufactures. Les vidéos sont réalisées selon un protocole précis et identique pour chacune :

- Le portrait de l'artiste,

- Le portrait du parrain,

- Les échanges entre l'artiste et son parrain,

- Les échanges entre l'artiste et les artisans,

- La présentation finale des oeuvres.

À la fin de chaque cycle de quatre ans, soit entre douze et quinze résidences selon les cycles, les travaux des artistes sont exposés dans un lieu prestigieux comme le Palais de Tokyo à la fin de l'année 2017 pour le cycle 2013-2016 accompagnés des vidéos. Le titre de l'exposition reprend le titre du cycle de résidence.

Les œuvres seront ensuite exposées dans 5 espaces d'exposition hors lieux de vente qu'Hermès possède dans le monde. (La Verrière à Bruxelles, Atelier Hermès à Séoul, Aloft at Hermès à Singapour, Le Forum à Tokyo ainsi que l'espace dédié aux expositions temporaires de La Grande Place musée du cristal Saint-Louis).

La présence dans les médias est essentiellement visible dans le secteur de la culture avec des reportages dans des revues spécialisées internationales : Intramuros, The Art News Paper, Wired, etc. Quand les résidences d'artistes sont évoquées dans la presse hors du secteur culturel, l'angle privilégié est celui de la Responsabilité Sociale et Environnementale (RSE).

\section{Productions d'oeuvres et de signes : les vidéos}

De la coopération à l'intérieur même des ateliers de fabrication entre artistes et artisans, naissent des objets uniques qui portent à la fois l'empreinte de l'artiste et l'identité de la marque tout en projetant sur la manufacture, l'aura d'un lieu de création. Les matériaux et les savoir-faire mis en œuvre sont identiques à ceux utilisés pour les produits destinés à la vente. Les créations de certains artistes alimentent ensuite l'inspiration des équipes de style pour les lignes de produits commerciales. Les vidéos donnent à voir un récit visuel et verbal plutôt stéréotypé conforme à l'image traditionnelle de la marque. 
La question de la nature artistique de la réalisation ne se pose pas en regard des pratiques de l'art contemporain qu'elle intègre a priori alors qu'elle se poserait si l'objet était considéré comme un prototype d'une série qui devrait réussir l'épreuve de l'artification a posteriori. L'installation «malle et accessoires » correspond parfaitement au critère incontournable du récit que l'œuvre contemporaine est censée véhiculer (N. Heinich, 2014, p. 89). Dans le cas du travail sur le tissu, l'œuvre surfaite du point de vue de la matière générée réside surtout dans l'installation pluridimensionnelle. C'est aussi une œuvre « relationnelle » (N. Bourriaud, 2001, p. 44) où on voit un tissu de relations intellectuelles se tramer entre l'artiste, le technicien et le parrain faisant aussi œuvre. Enfin les réalisations du verrier appartiennent plus au champ du design car il s'agit d'objets d'usage (vase, bougeoirs, saladier) même si la fonction n'est pas immédiatement perceptible sans explication.

Dans toutes les vidéos, l'architecture des lieux est simplement suggérée par des détails des ateliers où on ne voit que des opérations manuelles : un spectateur non averti peut penser que toute la production Hermès se déroule ainsi dans une ambiance artisanale intemporelle. Pour le tissage de soie où les métiers utilisés pour les coupes-types (tissages de quelques mètres) sont des métiers industriels, on ne voit à aucun moment la fabrication réelle de la pièce. Nous assistons simplement à une discussion avec le chef d'atelier autour d'échantillons. Les fours des verriers sont aussi des fours pour prototypes. La vivacité du feu impose un élément primaire vers lequel la caméra revient toujours. La scène de l'artiste et des artisans réunis autour du feu renvoie à une vision assez primitive.

Les outils montrés en très gros plan sont d'excellents véhicules visuels du savoir-faire en action : ils sont montrés polis par l'usage, impeccablement rangés, et relient la main du travailleur et l'œuvre. On voit l'alène pénétrer le cuir, le fil ciré passer et la canne de souffleur faire partie intégrante de l'identité professionnelle du verrier qu'il soit artisan de la manufacture ou artiste. Dans le cas du travail de la soie dont le procédé est plus industriel, l'absence des outils et des gestes du métier appauvrit l'image.

Le discours est à l'image de la marque, tout en retenue. Les échanges sont très éloignés de la réalité du travail de création que nous avons pu connaître et qui fait alterner moments d'exaltation, de tension, d'attention, de déception et de calme. Le même ton de voix monocorde est utilisé par les trois artistes et leurs trois parrains dans toutes les vidéos.

Les dialogues oscillent entre une simplicité presque caricaturale du fait de la répétition de termes populaires et un discours abscons dans le registre de l'art contemporain, un « art de l'anecdote» (N. Heinich, 2014, p. 90) : on parle de «réappropriation de l'aléatoire et du rapport au lieu», de «question beckettienne», des «choses qui adviennent dans 
l'engagement» ou des «structures molles». Ces expressions prononcées au milieu des artisans rappellent la distinction sociale entre l'artiste et l'artisan tandis que les relations humaines obéissent à des figures de style convenues : celle du maître (Jean-Michel Albérola), la figure de l'ingénue (Io Burgard), la complicité intellectuelle entre le professeur et l'artiste verrier tous deux américains, une relation plus conviviale et égalitaire entre Célia Gondol et sa marraine Ann Veronica Janssens. Les stéréotypes traditionnels restent très présents. La maîtrise du discours sur l'art contemporain dans ces vidéos évoque évidemment «le récit de l'œuvre constitutif du paradigme de l'art contemporain » (N. Heinich, 2014, Ibid.).

Les séquences de travail avec les artisans et les tâches accomplies en commun pendant la phase de réalisation témoignent d'une forme de compagnonnage entre artisan et artiste même si elles ne sont pas exemptes de clichés, avec des ouvrières en blouse, le tutoiement de rigueur, les gros plans sur les gestes. Les dimensions métaphoriques des processus de création sont évoquées plusieurs fois.

Cette expérience intimiste valorisant les artisans et leurs savoir-faire sur leur lieu de travail est une constante de la communication interne et externe d'Hermès, un film moyen métrage a déjà été dédié aux métiers manuels sous le titre «Les mains d'Hermès » (2011) : ils font partie du dispositif de patrimonialisation de la marque.

Discours et programmes

Dans le rapport d'activité 2016 de la fondation d'entreprise Hermès, son président déclare que son « objectif essentiel» est de « créer les conditions propices au développement d'un monde meilleur» et que son engagement constitue les bases d'une «philanthropie éthique, réelle, sincère et appliquée » d'une multinationale (qui pousse le souci du détail jusqu'à faire écrire les rubriques nécrologiques de ses dirigeants par son service de communication).

La fondation développe neuf programmes qui s'articulent autour des savoir-faire entre deux pôles : d'une part « savoir-faire et création » et d'autre part, "savoir-faire et transmission ». Ces programmes concernent quelquefois des pratiques éloignées des métiers de l'entreprise comme les arts de la scène mais sont plus fréquemment au cœur de métier de l'entreprise avec le Prix Émile Hermès pour le design ou Manufacto et l'Académie des savoirfaire pour la découverte et l'approfondissement des métiers artisanaux. La fondation soutient aussi différents organismes nationaux et internationaux qui agissent pour la valorisation des métiers manuels.

Lorsqu'on analyse les programmes, les savoir-faire d'Hermès sont omniprésents au moins de manière subliminale mais ses intérêts stratégiques aussi, comme on peut le voir dans les performances en soutien à des artistes étrangers en particulier asiatiques pour des expositions 
dans leur pays alors que l'Asie est le nouvel Eldorado du luxe. Le programme dédié à la photographie contemporaine s'articule autour d'opérations croisées entre artistes et curateurs français et américains sans oublier que les États-Unis sont un des principaux marchés de la maison Hermès. Les célèbres carrés (grands foulards en soie) de la marque imprimés d'oeuvres de photographes, un mécénat qui a un programme spécifique, sont proposés dans sa galerie en ligne et son lieu d'exposition de Bruxelles tandis que le nouveau programme destiné à revaloriser les métiers manuels auprès des plus jeunes est directement en rapport avec les besoins en ressources humaines de la maison de luxe. L'engagement en faveur de la planète à travers le programme Diversité prend en compte l'évolution de la législation concernant les espèces menacées dans une stratégie de dédiabolisation de l'utilisation des peaux rares, pratique incontournable dans le luxe pour les pièces uniques, qui pose un problème d'image à la marque. Ainsi la fondation d'entreprise reste très en phase avec les préoccupations stratégiques du groupe.

Confier la création d'une collection d'entreprise à des jeunes artistes revisitant des métiers anciens sous l'ombre tutélaire de parrains attentifs apparaît comme une métaphore de la marque, du groupe familial historique et des dirigeants successifs qui ont fait évoluer les produits et faciliter les passages de flambeaux successifs selon un rituel qui commence toujours par un passage symbolique dans un atelier.

On voit ainsi comment les créations des artistes en résidence dans les manufactures confèrent un caractère atypique à ce dispositif de collection d'entreprise en patrimonialisent des savoirfaire historiques dans des pièces incarnant le futur de la marque, non seulement dans leur dimension artistique contemporaine mais aussi dans le nomadisme hypermoderne de leur mise en exposition, alors que la collection d'entreprise se construit plus traditionnellement sur le récit historique de la maison et sur sa mémoire. Ce caractère atypique est renforcé par la conjugaison à chaque étape de la double temporalité de la maison que nous avons déjà évoquée à la fois ancrée dans son passé et projetant ses métiers dans l'avenir.

\section{Résidences d'artistes et transfiguration du banal}

Alors que la maison Hermès joue en permanence sur le statut «d'objet-frontière » entre produit et objet d'art, musée et boutique, expériences artistiques, design de produit et création, les réalisations des résidences d'artistes sont toutes immédiatement identifiées comme des oeuvres grâce à l'accumulation d'éléments de légitimation: auteurs issus d'écoles d'art reconnues, fabrication de pièces uniques, récit propre à l'art contemporain mais aussi du fait du dispositif en œuvre depuis la sélection des artistes jusqu'aux lieux d'exposition hors des lieux de vente. Ce résultat contraste avec les tentatives d'artification des produits de luxe qui 
échouent presque toujours leur passage à l'art c'est-à-dire à la transfiguration du banal conceptualisée par Arthur Danto qui « introduit les concepts de rhétorique, de style et d'expression dans l'espoir qu'ils nous permettraient de nous rapprocher davantage d'une définition de l'art...pour distinguer les œuvres d'art des autres supports de représentation qui leur ressemblent sans partager le statut » (Danto,1981, p. 261). Le dispositif rhétorique de la résidence d'artiste «amène les auditeurs d'un discours à adopter une attitude spécifique envers l'objet de celui-ci et de les amener à le voir dans une lumière particulière » (Danto, 1981, p. 262). En tant que style (au sens du mot dans le langage), le produit « œuvre d'art» apparaît comme une métaphore de la marque « dès lors que la manière dont un sujet est représenté est mise en relation avec le sujet représenté » (Danto, 1981, p. 306) c'est-à-dire dans le cas étudié, lorsque les modalités de mise en valeur du produit-œuvre sont objectivement reliées à la marque représentée par les matériaux utilisés ou la mise en scène de l'atelier. Ainsi du point de vue de la philosophie de l'art on peut estimer que la question de la frontière reste imprécise et aléatoire entre les produits de l'industrie du luxe et les objets de ce secteur en exposition muséale. Pour en juger in situ et concrètement, il est intéressant de passer un peu de temps dans les magasins Hermès de facture récente : celui de la rue de Sèvres à Paris établi dans l'ancienne piscine de l'hôtel Lutétia procède davantage de l'installation d'art contemporain notamment avec ces structures en bois délimitant des espaces d'exposition que de l'étalagisme, les deux dispositifs fusionnant selon Yves Michaud (2017).

\section{Conclusion}

Alors que la marque est imprégnée de l'épopée familiale et des sept générations qui se sont succédées à sa tête, les résidences d'artistes en entreprise actualisent le processus de patrimonialisation, en y injectant un ester, celui de «l'art à l'état gazeux » (Y. Michaud, 2003) qui est celui d'une «expérience esthétique diffuse et vaporeuse à contenu fluide et plaisant [sans questionnement philosophique]» (Ibid., p. 181). Elles affranchissent la valorisation du patrimoine des limites d'une collection d'entreprise tournée vers le seul récit historique.

Les œuvres restent chargées d'une dimension mémorielle rattachée à la personne même de l'artiste qui les a façonnés et s'intègrent à cette dynamique de collection grâce à une forme narrative hybride de passé, de présent et d'avenir dans un storytelling à la fois visuel et textuel. Luc Boltanski et Arnaud Esquerre critiquent cette approche qu'ils appellent la «patrimonialisation provoquée » (L. Boltanski \& A. Esquerre, 2014, p.11) mais identifient cependant l'intérêt de formes conventionnelles différentes en mettant l'accent sur les modalités de leur mise en valeur qui contribuerait à une construction sociale de la singularité. 
L'agencement technique autour de cette activité opère «dans un double jeu de la médiatisation et de la médiation » (J. Davallon, 1999, p. 36) tout en conservant une dimension conceptuelle.

Cette collection d'entreprise vit une reconfiguration permanente dans un nomadisme temporel et géographique : à l'image d'une pièce de cristal représentant la quintessence d'un savoirfaire ancien présenté dans le musée historique à côté du site de production puis dans une galerie Hermès ultra-contemporaine de Séoul avec projection des scènes de travail des artisans et d'un Art PhD dans une simplicité chorégraphiée. Il ne s'agit pas d'une initiative isolée comme on a pu le constater à travers l'implication croissante de l'industrie du luxe dans l'art contemporain: Yves Michaud dans une note de blog de 2017 qualifie ce système de « fait social de la société esthétisée [...] un ensemble de pratique et de comportements d'acteurs autour d'une machine de production, de marketing, de distribution et de vente. » Il relève la quasi-confusion entre le système de l'art contemporain et celui de l'industrie du luxe en déplorant « la production tranquille d'un non-style»(Y. Michaud, 2017) qui lui semble mortifère pour l'art, victime du triomphe de l'esthétique. On peut s'interroger si ces dispositifs de communication ne se déploient pas grâce à la dépossession des cadres culturels nécessaires à la compréhension de l'art au profit d'un regard plus superficiel sur un objet simplement arty. À l'ère du triomphe de l'esthétique ou plutôt de l'esthétisation, la collection d'entreprise comme l'art contemporain opèrent dans un processus de communication d'une expérience, un marquage identitaire : «il ne s'agit pas de montrer des chefs d'œuvre mais de marquer sa place, de se signaler et d'exister, y compris comme souvent, dans la fiction d'une identité reconstruite et réinventée » (Y. Michaud, 2003, p. 196).

Les résidences d'artistes de la maison Hermès reconfigurent par cycle la collection d'entreprise et permettent de générer des nouvelles interprétations de celle-ci : hybride et mobile moins intemporelle que conjuguant plusieurs temporalités dans un nomadisme muséal qui répond à l'hyper-modernité internationale du client nomade.

\section{Bibliographie}

Anderlini-Pillet V. (2016). L'artification du luxe dans le discours médiatique : des signes au sens (2010-2014) thèse de doctorat disponible à :

https://tel.archives-ouvertes.fr/tel-01492962/document

Anderlini-Pillet V. (2017) Conjuguer patrimonialisation et innovation dans le storytelling: l'affrontement de deux temporalités dans le luxe. Communications \& Organisations, Accélérations Temporelles. Paris : L’Harmattan. 2017, pp. 87-98. 
Boltanski, L., \& Esquerre, A. (2017). Enrichissement : une critique de la marchandise. Paris : Gallimard.

Boltanski, L., \& Esquerre, A. (2014). La « collection », une forme neuve du capitalisme, la mise en valeur économique du passé. Les Temps Modernes, nº67, pp.5-72.

Cousserand, I. (2009). Les musées d'entreprise, un genre composite. Communication et organisation, (35), pp.192-214.

Danto, A. C. (1980). La transfiguration du banal : une philosophie de l'art. Paris : Éditions du Seuil (1989 pour la traduction française).

Davallon, J. (1999) L'exposition à l'œuvre. Paris : L'Harmattan, collection Communication et civilisation.

De Ferrière Le Vayer, M. (2007). Des métiers d'art à l'industrie du luxe en France ou la victoire du marketing sur la création. Entreprises et histoires, (46), 157-176.

Fondation d'entreprise Hermès. (2016) Rapport d'activité. Téléchargeable à : http://www.fondationdentreprisehermes.org/La-Fondation

Fontagné L. (dir.) \&Groupe G-Mond PSE. (2014). Positionnement de gamme et compétitivité : les enseignements du secteur du luxe français. Paris : Comité Colbert. p.13.

Goodman, N. (1978). Manières de faire des mondes. Paris : Folio Essais.

Heinich, N., \& Shapiro, R. De l'artification: enquêtes sur le passage à l'art. (2012). Paris : Éditions de l'École des hautes études en sciences sociales.

Lipovetsky, G., \& Serroy, J. (2013). L'esthétisation du monde : vivre à l'âge du capitalisme artiste. Paris : Gallimard.

Michaud, Y. (2003). L'art à l'état gazeux essai sur le triomphe de l'esthétisme. [Paris] : A. Fayard. p.196

Michaud, Y, (2017). Quelques réflexions (de plus) sur l'art contemporain http://michaudphilo.blogspot.fr/2017/10/

Poulot, D. (2006). Une histoire du patrimoine en occident, $\mathrm{XVIII}^{\mathrm{e}}-\mathrm{XX}^{\mathrm{e}}$ siècle : du monument aux valeurs ( $1^{\text {re }}$ éd). Paris : Presses universitaires de France.

Ravix, J. T. (2013). Luxe, innovations et crise : les enseignements des économistes classiques. Innovations, (41), p. 29-49.

Iconographie

Les vidéos des résidences d'artistes sont disponibles à :

http://www.fondationdentreprisehermes.org/Savoir-faire-et-creation\#Residences-d-artistes

Les mains d'Hermès, (2011). le film est accessible à : https://vimeo.com/160364562 